\title{
O processo de urbanização na província de Córdoba, Argentina. Para uma empirização do tempo ${ }^{1}$
}

\author{
Urbanization processes in Córdoba province, Argentina. \\ Towards an "empirización" of the time
}

\author{
Franco Gastón Lucero \\ Maria de los Ángeles Galfioni \\ Mónica Donadoni \\ Analía Emiliozzi \\ Jorge Luis Hernández
}

Universidad Nacional de Río Cuarto Córdoba - UNRC - Río Cuarto - Córdoba - Argentina

\begin{abstract}
$\longrightarrow$
Resumo: A atual configuração territorial dos assentamentos populacionais urbanos da Argentina foi o resultado de um longo processo histórico de construção social, que tem respondido ao contexto político-econômico da história do país, fortemente influenciada pela sua inserção na divisão internacional do trabalho.As cidades, pontos nodais das economias internas, mostram uma estrutura e dinâmica populacional próprias, resultante do seu comportamento demográfico interno e sua relação sistêmica com outras unidades em assentamentos populacionais formando um sistema urbano provinciano solidário às estratégias nacionais.O objetivo deste trabalho éanalizar evolução e a distribuição da população e assentamentos urbanos na província de Córdoba e sua relação com os diferentes períodos da história socioeconômica e política da Argentina, tentando identificar alguns problemas emergentes dessa configuração.O período de interesse, entre 1869 e 2010, foi definido pela disponibilidade de censos nacionais que contribui com base empírica para esta proposta e os resultados são apresentados em termos de análise e reflexão dos indicadores sintéticos e expressões cartográficas.
\end{abstract}

Palavras-chave: Processo de Urbanização. Sistema Urbano. Contexto políticoeconômico.

Abstract: The current territorial configuration of settlements of the urban population in
Argentina has been the result of a long historical process of social construction. Such
process has resulted from the political-economic context in the country's history, which
was strongly influenced by its insertion in the international division of the labour.The
cities, nodal points of internal economies, have their own dynamics and population
structure, which are the result from their internal demographic movements and
systemic relationship with other units of population settlements, thus forming a
provincial urban system, which supports the national strategies. The aim of this work is
to study the evolution and distribution of the urban population and urban settlements in
the Córdoba province, attending at their evolution in relation to the different
socioeconomic periods in Argentina's political history, in order to identify some
emerging problems of that configuration. The period of interest lies between 1869 and
2010 , and is characterized by the availability of national censuses, which provide the
empirical support for this proposal. The results will be presented in terms of an analysis

${ }^{1}$ El presente trabajo se enmarca dentro del Proyecto de Investigación Orientado en Red (PIO) "Singularidades territoriales: tramas productivas, calidad de vida y vulnerabilidad. Líneas de acción para la reducción de las disparidades departamentales en la provincia de Córdoba" financiado por el Ministerio de Industria, Comercio, Minería y Desarrollo Científico y Tecnológico de la provincia de Córdoba. 
and reflection of the synthetic indicators and cartographic expressions derived from the study.

Keywords: Urbanization process. Urban system. Economic-political context.

\section{Introdução}

El territorio es resultado del ejercicio de relaciones sociales heterogéneas en un contexto témporo-espacial determinado (MANZANAL, 2007). Los intereses de los actores sociales involucrados se materializan en el espacio a través de acciones concretas que responden a patrones preestablecidos por el modelo socioeconómico vigente.

En este sentido, a partir de una primera aproximación a la lectura de los datos censales considerados entre 1869-2010, se evidencia que el proceso de urbanización alcanzado por las distintas provincias del país en los diferentes períodos de organización espacial ha sido heterogéneo, como consecuencia de un grado de desarrollo económico diferencial entre las distintas regiones del país, generando una desigual distribución de la población y de los asentamientos urbanos en el territorio.

Desde esta perspectiva, el presente trabajo se propone estudiar la evolución y distribución de la población y los asentamientos urbanos de la provincia de Córdoba y su vinculación con los diferentes períodos de la historia económica y política de la Argentina, entre 1869 y 2010, intentando comprender los profundos desequilibrios territoriales e identificar los problemas emergentes de esa configuración.

Si bien dentro de esta temática se registran antecedentes a nivel nacional y provincial, incluyendo algunos de los propios autores (HERNÁNDEZet al, 2010; HERNÁNDEZet al,2014), los mismos presentan un abordaje de la temática desde diferentes perspectivas y enfoques, que sirven de base para la consecución del objetivo del presente trabajo.

Para su ejecución, se realiza un tratamiento estadístico y cartográfico de los datos censales de la población, considerando el período considerado. Dicho corte temporal se fraccionan en tres segmentos, en función de los modelos socioeconómicos definidos en el proceso de inserción del país en la División Internacional de Trabajo (DIT), que se constituye como marco explicativo para comprender la dinámica poblacional urbana, como una forma de aproximación a una empirización del tiempo. Estos segmentos constituyen sistemas temporales que son analizados y apoyados en la utilización de indicadores estadísticos sintéticos y cartografía temática.

A continuación se discuten algunas consideraciones teóricas-metodológicas vinculadas al fenómeno de urbanización como proceso demográfico y económico-social. Luego, se presenta el proceso de generación de las disparidades observadas en los sistemas temporales identificados y finalmente, se exponen los principales hallazgos de este trabajo y algunas reflexiones a modo de conclusión.

\section{Consideraciones teóricas}

La relación espacio-tiempo y su unicidad como categoría de análisis, para la comprensión de los procesos que acontecen en el territorio, ha sido una de las grandes preocupaciones para los geógrafos y otros estudiosos en el devenir de la historia. Las discusiones teóricas con sus diferentes aportes han logrado superar las ambiguas consideraciones sobre dicha relación, alcanzando la noción de espacio geográfico como una totalidad concreta a partir de la comprensión dialéctica espacio-tiempo dada por la empirización del tiempo (SANTOS, 2000).

Respecto a esto último, Santos (2000) manifiesta que la mejor forma de unificar tiempo y espacio es empirizando el tiempo, ya que el espacio posee siempre una componente de materialidad desde donde emerge una parte de concreción y empiricidad. En este sentido, el punto de partida es la realización de la sociedad sobre la base material, 
tales como el espacio y su uso, el tiempo y su uso, la materialidad y sus diversas formas, las acciones y sus diversos aspectos. Por esta razón, el autor expresa que así empirizamos el tiempo, haciéndolo material y, de ese modo, lo asimilamos al espacio, que no existe sin la materialidad.

Este rasgo de unión dialéctico entre el espacio y el tiempo, es lo que define al espacio geográfico como totalidad que se metamorfosea en un conjunto indisoluble, solidario y contradictorio de sistemas de objetos y sistemas de acciones mediados por las normas (SANTOS, 2000). Dicha totalidad resulta entonces en una suspensión temporal y metodológica del proceso de totalización, el cual conduce de la antigua a la nueva totalidad y constituye la base de conocimiento de ambas (SANTOS, 2000). En este caso, los objetos, acciones y normas crean eventos y dichos acontecimientos ocurren en el tiempo y en el espacio constituyendo entonces el vehículo que imbrica a ambos planos de la realidad en una totalidad concreta.

Ahora bien, para la comprensión de esa totalidad resulta necesaria su escisión en varias totalidades en función de algunas variables de interés, con el objeto de analizar la estructura significativa del territorio a partir de su coherencia interna. Estas totalidades pueden ser también entendidas como sistemas temporales 0 periodizaciones (SANTOS, 2000). De todos modos, más allá de estos recortes temporales, la totalidad concreta se entenderá en su totalidad mayor.

Entendiendo al espacio geográfico como territorio usado, ocupado por una densa trama de relaciones materiales y sociales, asiento de recursos naturales y producidos, escenario de cooperación y disputa (SANTOS, 1994; SILVEIRA, 2010; 2013), el contenido y la dinámica territorial pueden ser "al mismo tiempo, condición y resultado producto de las divisiones territoriales del trabajo" (SILVEIRA, 2009, p. 436).

El escenario latinoamericano, y el argentino en particular, han evidenciado una articulación subordinada de sus territorios a la división de trabajo a escala global, generando un uso de los espacios subnacionales que les define o condiciona su trayectoria.

En este contexto, las variantes regulatorias con las que el territorio argentino fue definiendo su inserción a la DIT, son los eventos que caracterizan los diferentes modelos socioeconómicos, definiendo así los sistemas temporales que darán marco al análisis del proceso de urbanización en la provincia de Córdoba, Argentina.

Dicho proceso de urbanización no sólo involucra un aumento de la población urbana en relación a la población total, sino también implica cambios económicos, sociales y culturales. La morfología y trayectoria del sistema urbano son el lugar privilegiado de expresión de las adaptaciones territoriales a las variantes que ofrece la DIT.

\section{Uma breve digresión metodológica}

En el análisis empírico, para diferenciar población urbana de la rural se utiliza el umbral de tamaño que, para Argentina desde 1914, considera población urbana a las aglomeraciones de 2000 o más personas. Ese límite inferior y la observación de grandes concentraciones poblacionales, hacen conveniente la definición de diferentes categorías que expresen la diversidad de tamaños y que a la vez permitan hacerlas operativas. Para este trabajo se utiliza la propuesta de Vapnarsky y Gorjovsky (1990) (Tabla1), a la que se adicionó una subclasificación en la categoría de Pueblos grandes, a los fines de que sirvan como parámetro para comparar los censos de población y de tal manera ajustarla a la realidad poblacional de la provincia de Córdoba.

Tabla 1. Clasificación de aglomeraciones por tamaño poblacional

\begin{tabular}{|c|c|c|c|}
\hline & Categoría & Subcategorías & Población \\
\hline \multirow{6}{*}{ 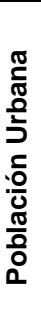 } & $\begin{array}{c}\text { III: Aglomeraciones } \\
\text { de más de } 1 \text { millón } \\
\text { de hab. }\end{array}$ & & Más de 1 millón \\
\hline & \multirow{2}{*}{$\begin{array}{c}\text { II: Aglomeraciones } \\
\text { de Tamaño } \\
\text { Intermedio (ATIs) }\end{array}$} & ATIs Mayores & 400.000 a 999.999 \\
\hline & & ATIs Menores & 50.000 a 399.999 \\
\hline & \multirow{3}{*}{$\begin{array}{l}\text { I: Ciudades y } \\
\text { pueblos }\end{array}$} & $\begin{array}{l}\text { Ciudades } \\
\text { Pequeñas }\end{array}$ & 20.000 a 49.999 \\
\hline & & \multirow{2}{*}{ Pueblos grandes } & 10.000 a 19.999 \\
\hline & & & 2000 a 9999 \\
\hline \multirow{2}{*}{\multicolumn{2}{|c|}{ Población Rural }} & Pueblos pequeños & Hasta 1999 \\
\hline & & $\begin{array}{l}\text { Población } \\
\text { Dispersa }\end{array}$ & $\begin{array}{ll}\text { Dispersa } & \text { en } \\
\text { campo abierto } & \end{array}$ \\
\hline
\end{tabular}

Fuente: Adaptación de Vapnarsky y Gorjovsky (1990). 


\section{Fundamento de los sistemas temporales}

El criterio de la escisión del tiempo histórico está definido, como se señaló anteriormente, por las variantes regulatorias que adaptan el territorio a los requerimientos de la DIT. En tal sentido, se presenta una somera caracterización de los modelos socioeconómicos a través de tres cortes temporales que son de utilidad para comprender la dinámica de la poblaciónurbana.

El primer período, identificado como Modelo Agroexportador, recorre en este trabajo de1869 a 1930 y se caracteriza por la definitiva ruptura del régimen colonial, la consolidación del Estado-Nación y la intensa liberalización del comercio internacional.

El sector dominante estaba conformado por una oligarquía terrateniente que permitió llevar adelante un proceso que marca la inserción de Argentina, como país proveedor de materias primas y alimentos, a los mercados internacionales asociados al capitalismo industrial europeo. En este período se produce un importante desplazamiento de la frontera agropecuaria y una profunda transformación del sistema urbano, fundamentalmente en la región pampeana, modelada por la creación de nuevas infraestructuras (redes ferroviarias) que la articula al puerto de Buenos Aires y más tardíamente a otros centros urbanos extra pampeanos (RAPOPORT, 2000; BASUALDO, 2006).

El segundo período, entre 1930 y 1975 , denominado Industrialización Sustitutiva de Importaciones (ISI), tiene su inicio en la fuerte restricción de los flujos comerciales emergentes de la crisis internacional de la década del '30 y los conflictos bélicos que constituyeron la base del anterior modelo socio-económico (AROSTEGUI et al, 2001).

Este período se caracteriza por un cambio regulatorio significativo, con un marcado avance de la intervención del Estado, la emergencia de una burguesía industrial local articulada con el capital extranjero y una notable redistribución de los ingresos, con transferencias desde el sector agropecuario al industrial y del capital al trabajo (BELINI y COROL, 2012).

El sistema urbano tiende a fortalecer a través de migraciones internas a los grandes aglomerados, espacios dinámicos del proceso de industrialización, potenciando las primacías emergentes del período anterior.

Por último, el tercer período, entre mediados de los años '70 y fines del Siglo XX, es el escenario de una nueva liberalización y desregulación económica. Las crisis del sistema monetario internacional y la del petróleo, permitieron al capital financiero hegemonizar el ordenamiento económico global forzando la reapertura de los mercados nacionales y dando inicio a un proceso de liberalización, desregulación y privatización, que van a tener un fuerte impacto en las estructuras productivas de Argentina, con un alto costo en términos de producción y empleo, y con escaso impacto sobre la urbanización definida en los períodos anteriores (BEJAR, 2011; FERRER, 2010).

Si bien en la primera década de este siglo algunos países latinoamericanos, incluyendo a Argentina, evidencian cambios en los regímenes regulatorios, no se registran efectos destacables sobre el sistema urbano de la nación ni de la provincia de Córdoba.

En síntesis, las diferentes estrategias de producción desarrolladas en el marco de los modelos socioeconómicos a escala nacional, en relación a escala mundial,han forjado la revalorización de algunos espacios funcionales al sistema mientras que otros quedan relegados, generando desequilibrios territoriales en términos de ocupación y distribución de la población y de los asentamientos urbanos. Estos últimos, en su constitución inicial van perfilando un conjunto de rasgos identitarios definidos por el rol específico que cumple en el contexto político, económico y social en el que emerge. Esa marca fundacional, se va modificando con el tiempo como respuesta a las transformaciones de su entorno, generando un proceso de adaptación muy dinámico en el que las ciudades van incorporando nuevas funciones (FORMIGA y PRIETA, 2011). 
5 El proceso de urbanización a escala provincial y su inserción en el contexto nacional

El crecimiento poblacional y la urbanización de la provincia de Córdoba, como del resto de los territorios subnacionales argentinos, han estado fuertemente influidos por las estrategias de integración en la DIT definidas a la escala nacional.

Una primera evaluación estática comparativa permite observar que, si bien el crecimiento poblacional cordobés acompañó la tendencia nacional, su ritmo más lento condujo a una pérdida de participación en el total, aunque la tasa de urbanización registró un crecimiento más acelerado aproximándose a los valores nacionales.

Tabla 2. Crecimiento poblacional y tasa de urbanización por períodos entre 1869 y 2010. Argentina y Córdoba.

\begin{tabular}{|c|c|c|c|}
\hline \multirow[b]{2}{*}{$\begin{array}{l}\text { Crecimiento de } \\
\text { la Población }\end{array}$} & \multicolumn{3}{|c|}{ Período 1869-1930 } \\
\hline & $\begin{array}{c}\text { Tasa Anual } \\
\text { de } \\
\text { crecimiento }\end{array}$ & $\begin{array}{c}\text { Participación } \\
\text { porcentual } \\
\text { del } \\
\text { crecimiento }\end{array}$ & $\begin{array}{c}\text { Tasa de } \\
\text { urbanización } \\
\text { (1947) }\end{array}$ \\
\hline Total del país & $3,02 \%$ & $100,00 \%$ & $62,50 \%$ \\
\hline Córdoba & $2,56 \%$ & $8,90 \%$ & $50,50 \%$ \\
\hline \multirow[b]{2}{*}{$\begin{array}{l}\text { Crecimiento de } \\
\text { la Población }\end{array}$} & \multicolumn{3}{|c|}{ Período 1869-1930 } \\
\hline & $\begin{array}{c}\text { Tasa Anual } \\
\text { de } \\
\text { crecimiento }\end{array}$ & $\begin{array}{c}\text { Participación } \\
\text { porcentual } \\
\text { del } \\
\text { crecimiento }\end{array}$ & $\begin{array}{c}\text { Tasa de } \\
\text { urbanización } \\
\text { (1947) }\end{array}$ \\
\hline Total del país & $1,87 \%$ & $100,00 \%$ & $83,00 \%$ \\
\hline Córdoba & $1,73 \%$ & $8,20 \%$ & $80,70 \%$ \\
\hline \multirow[b]{2}{*}{$\begin{array}{l}\text { Crecimiento de } \\
\text { la Población }\end{array}$} & \multicolumn{3}{|c|}{ Período 1869-1930 } \\
\hline & $\begin{array}{c}\text { Tasa Anual } \\
\text { de } \\
\text { crecimiento }\end{array}$ & $\begin{array}{c}\text { Participación } \\
\text { porcentual } \\
\text { del } \\
\text { crecimiento }\end{array}$ & $\begin{array}{c}\text { Tasa de } \\
\text { urbanización } \\
\text { (1947) }\end{array}$ \\
\hline Total del país & $1,30 \%$ & $100,00 \%$ & $91,00 \%$ \\
\hline Córdoba & $1,14 \%$ & $7,40 \%$ & $89,70 \%$ \\
\hline
\end{tabular}

Fuente: Elaboración propia en base a datos de los Censos Nacionales de Población y Vivienda de 1869, 1895, 1914, 1947, 1960, 1970, 1980, 1991, 2001 y 2010. INDEC-Argentina.

Buenos Aires, Santa Fe, Entre Ríos y Catamarca fueron las que presentaron las mayores tasas de urbanización en el primer censo nacional de población de 1869, manteniéndose las dos primeras hasta la actualidad. La provincia de Catamarca, de fuerte desarrollo durante la colonización española, sufrió un proceso de marcada ruralización de su población mostrando, aún en la actualidad, valores de urbanización muy reducidos en el contexto nacional.

Las provincias patagónicas, casi carentes de sujetos censales ${ }^{2}$ en los primeros relevamientos, presentan en la actualidad niveles de urbanización entre los más elevados, como resultado de las estrategias de ocupación del espacio nacional, que matizaron las tendencias espontáneas inducidas por los cambios en la matriz productiva derivada de la inserción nacional en la DIT.

La dinámica del uso del territorio cordobés se presenta, de manera estilizada, en la Tabla3. En ella se describen los diferentes ritmos de crecimiento de la población total a nivel provincial y departamental y sus impactos sobre la urbanización como expresión de la respuesta territorial a los cambios en los marcos regulatorios nacionales que definen los sistemas temporales seleccionados en el presente trabajo.

\footnotetext{
${ }^{2}$ Los naturales de pueblos originarios no se registraron en los primeros censos.
} 
Tabla 3. Crecimiento poblacional y tasa de urbanización por períodos entre 1869 y 2010 a escala departamental en la provincia de Córdoba

\begin{tabular}{|c|c|c|c|c|c|c|c|c|c|}
\hline \multirow[b]{2}{*}{\begin{tabular}{c} 
Departamentos de la Provincia de Córdoba \} $\\
{\text { Población }}$ & \multicolumn{3}{|c|}{ 1. Período 1869 - 1930} & \multicolumn{3}{|c|}{ 2. Período 1930 - 1975} & \multicolumn{3}{|c|}{ 2. Período $1975-2010$} \\
\hline & $\begin{array}{c}\text { Tasa Anual de } \\
\text { Crecimiento }\end{array}$ & $\begin{array}{l}\text { Participación } \\
\text { Porcentual del } \\
\text { Crecimiento }\end{array}$ & \begin{tabular}{|c|} 
Tasa de \\
Urbanización \\
(1947)
\end{tabular} & $\begin{array}{l}\text { Tasa Anual de } \\
\text { Crecimiento }\end{array}$ & $\begin{array}{c}\text { Participación } \\
\text { Porcentual del } \\
\text { Crecimiento }\end{array}$ & $\begin{array}{c}\text { Tasa de } \\
\text { Urbanización } \\
(1980)\end{array}$ & $\begin{array}{c}\text { Tasa Anual de } \\
\text { Crecimiento }\end{array}$ & \begin{tabular}{|c|} 
Participación \\
Porcentual del \\
Crecimiento
\end{tabular} & $\begin{array}{c}\text { Tasa de } \\
\text { Urbanización } \\
\text { (2010) }\end{array}$ \\
\hline 1. Calamuchita & 1,10 & 1,08 & $s / d$ & 1,41 & 1,31 & 56,2 & 1,41 & 1,95 & 74,3 \\
\hline 2. Capital (ex Ciudad) & 3,12 & 23,57 & 95,6 & 3,11 & 55,61 & 97,7 & 1,15 & 40,50 & 99,1 \\
\hline 3. Colón (ex Anejo Norte hasta 1892) & 2,73 & 2,79 & 41,6 & 2,42 & 4,53 & 70,7 & 2,92 & 13,18 & 91,3 \\
\hline 4. Cruz del Eje & 1,47 & 2,17 & 49,5 & 0,99 & 1,39 & 65,4 & 0,68 & 1,14 & 72,8 \\
\hline 5. Gral. Roca (ex Río Cuarto desde 1872 hasta 1888) & 5,28 & 3,42 & 22,2 & $-0,02$ & $-0,02$ & 55,3 & 0,62 & 0,64 & 73,5 \\
\hline 6. Gral. San Martín (ex Tercero Abajo hasta 1946) & 3,17 & 4,65 & 57,1 & 1,56 & 3,71 & 77,3 & 1,05 & 3,59 & 88,2 \\
\hline 7. Ischilín & 0,48 & 0,56 & 64,4 & 0,81 & 0,66 & 75,2 & 0,55 & 0,50 & 80,8 \\
\hline 8. Juárez Celman (ex Río Cuarto hasta 1888) & 4,59 & 4,27 & 36,1 & 0,39 & 0,60 & 63,3 & 0,94 & 1,57 & 78,9 \\
\hline 9. Marcos Juárez (ex Tercero Abajo hasta 1888) & 3,25 & 7,13 & 42,6 & 0,69 & 2,04 & 79,4 & 0,37 & 1,17 & 91,4 \\
\hline 10. Minas & $-0,23$ & $-0,13$ & 0,00 & $-0,67$ & $-0,15$ & 0,00 & $-0,28$ & $-0,04$ & 0,00 \\
\hline 11. Pocho & 0,16 & 0,08 & 0,00 & $-0,30$ & $-0,07$ & 0,00 & $-0,28$ & $-0,05$ & 0,00 \\
\hline 12. Pte. Roque Sáenz Peña (desde 1937) & 4,36 & 2,20 & 40,0 & 1,09 & 1,00 & 63,8 & 0,44 & 0,47 & 81 \\
\hline 13. Punilla & 2,20 & 2,33 & 43,6 & 2,75 & 5,13 & 88,7 & 2,07 & 8,42 & 97,3 \\
\hline 14. Río Cuarto (ex Gran Río Cuarto hasta 1888) & 4,36 & 9,17 & 48,8 & 1,81 & 8,31 & 78,6 & 0,91 & 6,17 & 91,2 \\
\hline 15. Río Primero & 1,46 & 2,62 & 6,6 & $-0,09$ & $-0,11$ & 29,8 & 0,86 & 1,11 & 68,7 \\
\hline 16. Río Seco & 1,09 & 0,58 & 0,0 & $-0,51$ & $-0,17$ & 0,00 & 1,56 & 0,51 & 53,8 \\
\hline 17. Río Segundo & 2,43 & 4,64 & 35,9 & 0,81 & 1,79 & 69,8 & 1,12 & 3,10 & 84,4 \\
\hline 18. San Alberto & 0,75 & 0,73 & 0,00 & 0,63 & 0,44 & 38,8 & 1,61 & 1,46 & 63,3 \\
\hline 19. San Javier & 1,04 & 1,42 & 43,1 & 0,77 & 0,84 & 59,1 & 1,26 & 1,75 & 71,5 \\
\hline 20. San Justo & 4,68 & 9,78 & 43,6 & 1,34 & 5,78 & 71,6 & 0,85 & 4,89 & 80,7 \\
\hline 21. Santa María (ex Anejo Sur hasta 1892) & 2,25 & 2,56 & 32,6 & 1,66 & 2,56 & 64,8 & 1,49 & 3,66 & 75,2 \\
\hline 22. Sobremonte & $-0,21$ & $-0,09$ & 0,00 & $-0,22$ & $-0,04$ & 0,00 & $-0,17$ & $-0,03$ & 49,1 \\
\hline 23. Tercero Arriba & 3,45 & 4,48 & 0,00 & 1,62 & 3,66 & 77,7 & 0,72 & 2,24 & 88,9 \\
\hline 24. Totoral & 1,06 & 0,78 & 0,00 & $-0,05$ & $-0,02$ & 30,97 & 1,03 & 0,51 & 44,25 \\
\hline 25. Tulumba & 1,34 & 1,09 & 0,00 & $-0,53$ & $-0,28$ & 0,00 & 0,03 & 0,01 & 35,3 \\
\hline 26. Unión (ex Tecero Abajo hasta 1860) & 4,42 & 8,12 & 43,3 & 0,51 & 1,51 & 66,3 & 0,50 & 1,57 & 80,1 \\
\hline \begin{tabular}{|c|} 
TOTAL \\
\end{tabular} & 2,56 & 100 & 50,5 & 1,73 & 100 & 80,7 & 1,14 & 100 & 89,7 \\
\hline
\end{tabular}}
\end{tabular}

Fuente: Elaboración propia en base a datos de los Censos Nacionales de Población y Viviendade 1869, 1895, 1914, 1947, 1960, 1970, 1980, 1991, 2001 y 2010. INDEC-Argentina.

En el período entre 1869-1930, se registra el proceso de crecimiento total de la población más acelerado delos tres en consideración. Si bien la provincia de Córdoba lo hizo a una tasa inferior a la del total nacional (tabla 2), se registran crecimientos muy relevantes en algunos de sus departamentos, entre los que se destacan los del sudeste provincial, de gran aptitud para la producción agropecuaria y fuertemente disputados a los pueblos originarios. La necesidad de ampliar el espacio productivo y el corrimiento de la frontera tuvieron un rol económico decisivo para esta región en el marco de la inserción subordinada a la DIT(EMILIOZZlet al, 2014).A partir de1880 los departamentos de las zonas Este y Sur también fueron receptores de una importante cantidad de inmigrantes en el marco de las políticas de colonización de tierras y de inmigración. Como contraparte, se observa un crecimiento muy lento, y aún decrecimiento, en departamentos muy activos en el periodo colonial.

Estos comportamientos antagónicos son muy elocuentes con relación a los ajustes poblacionales como respuesta a los cambios inducidos por el paso del régimen colonial a la inserción en la DIT que definió el Modelo Agroexportador.
Si bien en todos los sistemas temporales analizados se evidencia una correlación positiva entre la tasa de crecimiento anual media con el nivel de urbanización alcanzado al final del período, en esta etapa la intensidad de esa correlación es la más débil. El $71,22 \%$ del crecimiento poblacional se asentó en departamentos con tasas de urbanización inferiores a la del total provincial, reforzando la evidencia de la importancia relativa del sector agropecuario en esta etapa.

El registro de la tasa de urbanización y la participación en el crecimiento del departamento Capital ya ilustran la importancia de la ciudad de Córdoba como centro de atracción poblacional, por su desarrollo industrial, comercial y financiero (TERZAGA, 1963). Sin embargo, serán las condiciones del próximo sistema temporal las que consolidarán y ampliarán la primacía de ese aglomerado urbano.

El sistema temporal definido por el Modelo de Industrialización por Sustitución de Importaciones, entre los años 1930-1975, presenta una notable disminución del ritmo de crecimiento anual de la población en casi la totalidad de los departamentos de la provincia de Córdoba, concomitante con un 
intenso incremento en la tasa de urbanización, determinando en este período la correlación más intensa entre crecimiento y urbanización, muy característica de los procesos de industrialización.

En esta etapa, el 60,74\% del crecimiento fue absorbido por departamentos con tasas de urbanización superiores al total provincial, de los cuales el 55,61\% fueron al departamento Capital, definiendo una urbanización casi total de su espacio territorial. En tanto, los 22 departamentos de bajo nivel de urbanización explicaron sólo el $26,41 \%$ de ese crecimiento.

De los 11 Departamentos que crecieron más que la media en el período anterior, sólo tres -Capital, Colón y Río Cuarto- repitieron esa performance. Colón comenzando un proceso de aglomeración con la ciudad de Córdoba y Río Cuarto, concentrando la población del Sur provincial y consolidando su fuerte primacía en la ciudad homónima, cabecera departamental.

El tercer sistema temporal, período de vulnerabilidad financiera, entre los años 1975-2010, caracterizado por la reapertura al comercio internacional, la desregulación interna y la privatización de empresas del Estado, consolidó el sistema urbano provincial en los términos definidos en el período precedente.

Casi el $70 \%$ del crecimiento de este período fue absorbido por departamentos con tasas de urbanización más elevadas que el registro del total provincial. El ritmo de crecimiento fue más intenso en los departamentos Punilla y Colón, que se aglomeran en el Gran Córdoba, y en los de las serranías próximas a la Capital. En tanto, Río Cuarto registra en este período, por primera vez, una tasa de crecimiento inferior al promedio provincial.

A bien a diferencia del período anterior, donde la industrialización explicaba la urbanización creciente, en este período el fortalecimiento de la urbanización en presencia de desindustrialización ha sido explicado por la probabilidad de encontrar "empleos refugio", de baja productividad e ingresos, en los territorios más densamente poblados.

\section{Evolución y distribución de los asentamientos urbanos en la provincia de córdoba}

Tomando como base el análisis realizado en el apartado anterior, en el presente acápite se analiza el proceso de distribución de la población en asentamientos urbanos por categoría de tamaño poblacional, en base a los criterios definidos en la Tabla 1, con el propósito de precisar el fenómeno de urbanizacion particular de cada departamento.

La Figura 1 presenta tres cortes temporales que ilustran la situación al comienzo, la mitad y el final del período relevado por los Censos Nacionales.

\section{Figura 1}

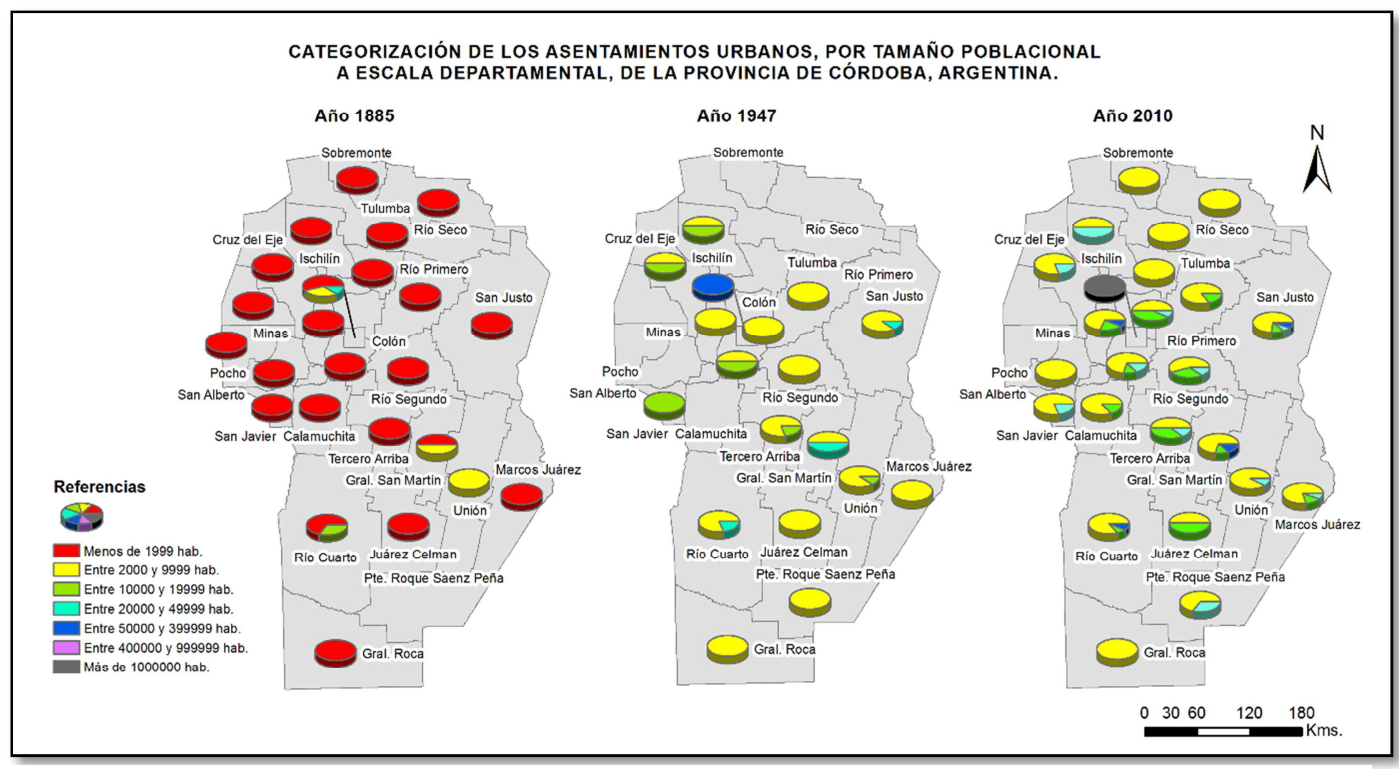

Fuente: Elaboración propia en base a datos del Censo Nacional de Población y Vivienda, INDECAraentina.

Ágora [ISSN 1982-6737]. Santa Cruz do Sul, v. 17, n. 1, p. 03-10, jan./jun. 2015. http://online.unisc.br/seer/index.php/agora/index 
El primero, de 1895, permite observar que la mayoría de los departamentos de la provincia de Córdoba contaba sólo con pueblos pequeños (menos de 2.000 habitantes). Sólo se registran aglomerados mayores en los departamentos Capital, General San Martín, Unión y Río Cuarto, evidenciando la fuerte ruralidad de los 220.022 pobladores cordobeses registrados, de los cuales el 13\% ya eran habitantes urbanos del departamento Capital.

El Censo Nacional de Población y Viviendade 1947 permite observar el resultado sobre la urbanización de la elevada tasa de crecimiento poblacional observada durante el Modelo Agroexportador. En tal sentido, se observa el incremento de centros urbanos en la mayoría de los departamentos, a excepción de los casos de Sobremonte, Río Seco, Tulumba, Pocho, Minas, San Alberto y Calamuchita.De los departamentos que contienen población urbana, en la mayoría predominan pueblos grandes, con dominancia de los de entre 2.000 y 10.000 habitantes. En varios departamentos emergen ciudades pequeñas como en el caso de Río Cuarto, con la ciudad homónima, San Justo con la ciudad de San Francisco y General San Martín con la de Villa María. Finalmente, se destaca la situación del departamento Capital con el predominio de ATIs mayor que aglomera un cuarto de las casi 1,5 millones de almas censadas.

Para el caso del año 2010, recogiendo el impulso urbanizador del período ISI, si bien se identifica una predominancia de departamentos con pueblos grandes, se observa además, una mayor heterogeneidad de distintas subcategorías urbanas y la emergencia y consolidación de ATIs. Así, las ciudades de Río Cuarto, Villa María y San Francisco incrementan su tamaño modificando su categoría, a la vez que, en el departamento Punilla, comienza a emerger como ATIs la ciudad de Carlos Paz.

El departamento Capital, completamente urbanizado, concentra el $40 \%$ de los 3,3 millones de personas censadas, que aumenta si se considera la aglomeración que se registra con localidades de los departamentos Colón y Punilla.

Esta dinámica poblacional ha generado una distribución que presenta concentraciones y vacíos poblacionales de magnitudes notables.

La Figura 2, que sintetiza esta situación, permite observar altas densidades poblacionales en el departamento Capital y sus alrededores, perdiendo intensidad hacia el norte, el sur y parte del oeste provincial. El departamento Río Cuarto, que aparece con mayor densidad que sus vecinos, debe esta situación a la alta concentración en la ciudad cabecera y se evidencia en el elevado valor del Índice de Primacía.

\section{Figura 2}

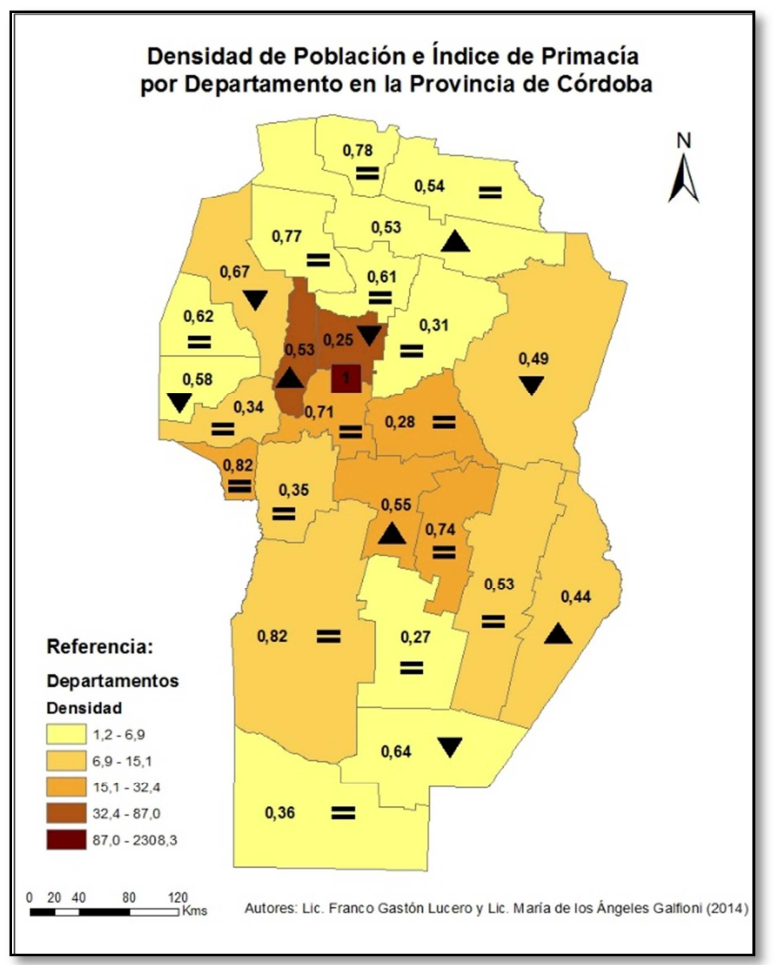

Fuente: Elaboración propia en base a los datos proporcionados por el Censo Nacional de Población y Vivienda del 2010. INDEC-Argentina.

Los departamentos del Centro-Este cordobés, presenta una mayor densidad que el Sur y, aunque incluye las ciudades de Villa María y San Francisco, los valores del Índice de Primacía indican una distribución por tamaño de aglomerados urbanos menos desigual.

Los departamentos Colón y Punilla, con elevada densidad y reducida primacía son el 
resultado de pueblos y ciudades pequeñas que se aglomeran a la capital provincial. (HERNÁNDEZet al, 2010).

En los departamentos del Noroeste de la provincia,los valores del Índice de Primacía son relativamente altos mientras que la densidad de población es baja, reflejando la herencia urbana del período colonial, ha sostenido algunos centros urbanos en medio de la pérdida de centralidad económica y población. Este grupo está integrado por los departamentos Sobremonte, Tulumba, Río Seco, Totoral, Ischilín, Cruz del Eje, Minas, Pocho y San Alberto.

El análisis de la evolución del Índice de Primacía entre 1895 y 2010, se puede observar que la mayoría (16 departamentos) manifiesta un comportamiento relativamente igual en todo el período sin tendencias hacia la concentración o desconcentración del centro urbano más primado. De los 8 departamentos restantes, 4 de ellos, Cruz del Eje, Colón, San Justo y Presidente Roque Sáenz Peña, presentan una desconcentración de la urbe más poblada, y otros 4, Tulumba, Punilla, Tercero Arriba y Marcos Juárez, expresan una tendencia evolutiva hacia la concentración de la población por parte de la ciudad de mayor tamaño.

\section{Considerações finais}

El proceso de urbanización en la provincia de Córdoba es expresión y materialización concreta de las racionalidades que guían las acciones e intenciones de las relaciones sociales que construyen el territorio en la totalidad temporal, en este trabajo definida por la inserción subordinada del territorio argentino a la división internacional del trabajo. Los sistemas temporales han sido delimitados en función de los diversos marcos regulatorios, eventos en término de Santos (2000), que dieron fundamento a los sistemas socioeconómicos particulares (Modelo Agroexportador, Industrialización por Sustitución de Importaciones y el período de Vulnerabilidad Financiera) y son la expresión particular de la empirización del tiempo.
La evolución de la urbanización en la provincia de Córdoba, como resultado concreto del proceso histórico, ha resultado en un territorio con marcadas disparidades que ha ido mutando como respuesta a las condiciones particulares de cada sistema temporal.

En el caso del Modelo Agroexportador ocupó, en términos censales, el Sureste cordobés. Por su parte, el ISI fortaleció las polarizaciones y las primacías que se reforzaron, a posteriori, en el período de Vulnerabilidad Financiera. La dinámica observada en los dos períodosprimeros, son previsibles en términos de las estrategias de ocupación. El período de inserción a la DIT como proveedor de materias primas y alimentos permitía prever la ocupación de los territorios aptos para esa producción. El proceso de industrialización se favoreció por economías de aglomeración potenciando la consolidación de grandes centros urbanos, el Gran Córdoba por ejemplo. Y en el caso del último período, menos previsible, se incrementó la urbanización y se mantuvieron o reforzaron las primacías como respuesta a la reestructuración productiva (desindustrialización).

Esta trayectoria ha definido un escenario de marcada polarización entre territorios fuertemente concentrados y espacios de muy baja densidad, generando importantes desafíos que interpelan las construcciones teóricas, empíricas y políticas entre las que destacamos: ¿Qué hacer con aquellas regiones marginadas estructuralmente por el sistema económico hegemónico? ¿Cuáles son las alternativas a desarrollar para atenuar los contrastes demográficos en la provincia de Córdoba? ¿Cómo contribuir con políticas sólidas y sustentables en el tiempo? Son algunos de los interrogantes que guiarán las acciones de nuestros futuros esfuerzos.

\section{Referências}

AROSTEGUI, J.; BUCHRUCKER, C. y SABORIDO, J. El mundo contemporáneo: Historia y problemas Madrid: Biblos-Critica, 2001. 958 p.

BASUALDO, E. Estudios de Historia Económica Argentina (desde mediados del siglo XX hasta la 
actualidad). Buenos Aires: Siglo XXI, FLACSO, 2006 , $496 \mathrm{p}$.

BEJAR, M. Historia del siglo XX. Europa, América, Asia, África y Oceanía. Buenos Aires: Siglo XXI Editores, 2011, $462 \mathrm{p}$.

BELINI, C. y KOROL J. C. Historia Económica Argentina del siglo XX. Buenos Aires: Siglo XXI, 2012, $312 \mathrm{p}$.

EMILIOZZI, A; DONADONI, M.; HERNÁNDEZ, J. y CASTRO, Mónica. Territorio y poder. Evolución de las divisiones político-administrativas en la provincia de Córdoba-Argentina. En: VIII JORNADAS DE INVESTIGADORES EN ECONOMÍAS REGIONALES,2014, Posadas, Misiones, p. 1-25.

FERRER, A. La Economía Argentina. Desde sus orígenes hasta principios del Siglo XXI. Buenos Aires: Fondo de Cultura Económica, 2010, 486 p.

FORMIGA, N. y PRIETO, M. B. Dinámica demográfica en ciudades intermedias. El caso de Bahía Blanca, Gran Río Cuarto y Neuquén-PlottierCipolletti. En: GORENSTEIN, S.; HERNÁNDEZ, J. y LANDRISCINI, G. (comp.). Economía urbana y ciudades intermedias. Trayectorias pampeanas $y$ norpatagónicas. Buenos Aires: CICCUS, 2011, p. 4783.

INSTITUTO NACIONAL DE ESTADÍSTICAS Y CENSOS -INDEC-. (2013) Censos Nacionales de Población y Vivienda de 1869, 1895, 1914, 1947, 1960, 1970, 1980, 1991, 2001 y 2010. Argentina.

HERNÁNDEZ, J.; DONADONI, M. y EMILIOZZI, A. Evolución del sistema urbano argentino. Una mirada a través de los censos nacionales de población. En: XXII JORNADAS DE HISTORIA ECONÓMICA, 2010, Río Cuarto, Córdoba, p.1-18.

HERNÁNDEZ, J.; GIAYETTO, J.; NATALI, P.; GALFIONI, M. A.; DONADONI, M.; EMILIOZZI, A. y LUCERO, F. Población y Territorio. Evolución de los asentamientos poblacionales y los sistemas productivos territoriales en la provincia de Córdoba (Argentina). En: XIII SEMINÁRIO INTERNACIONAL DA REDE IBEROAMERICANA DE INVESTIGADORES SOBRE GLOBALIZACIÓN Y TERRITORIO, 13, 2014, Salvador de Bahía, Brasil, p. $1-20$.

MANZANAL, M. Territorio, poder e instituciones. Una perspectiva crítica sobre la producción del territorio. En: MANZANAL, M; ARZENO, M. y NUSSBAUMER, B. (comp.). Territorios en construcción. Buenos Aires: CICCUS, 2007, p. 15-50.

SANTOS, M. Técnica, Espaço, Tempo. Globalização e Meio Técnico-Científico Informacional. São Paulo: Hucitec, 1994, 94 p.

SANTOS, M. (1996). A natureza do espaço. Técnica e tempo. Razão e emoção. São Paulo.Trad. a cargo de María Laura Silveira. La Naturaleza del Espacio.
Técnica y Tiempo. Razón y Emoción. Barcelona: Ariel, 2000, 348 p.

SILVEIRA, M. L. Región y División Territorial del Trabajo: Desafios en el período de la globalización. En: Revista Investigación y desarrollo, 17, 2, 2009, Colombia, p. 434-455.

SILVEIRA, M. L. Espaço geográfico e fenômeno técnico: por um debate substantivo. En: BOMFIM, P. R. A y SOUSA NETO, M. (Eds.). En: Geografia e Pensamento Geográfico no Brasil. São Paulo: Annablume, 2010, p. 123-139.

SILVEIRA, M. L. Tiempo y espacio en geografía: dilemas y reflexiones. En: Revista de Geografía Norte Grande, 54, 2013, Chile, p. 9-29.

RAPOPORT, M. Historia económica política y social de la Argentina. 1880-2000. Buenos Aires: Macchi, 2000, $1148 \mathrm{p}$.

TERZAGA, A. (1963) Geografía de Córdoba. Córdoba: Assandri, 1963, 364 p. VAPNARSKY, C. y GOROJOVSKY, N. (1990) El crecimiento urbano en la Argentina. Buenos Aires: Grupo Editor Latinoamericano, Instituto Internacional de medio Ambiente y Desarrollo-IIED, 1990,160p. 